\title{
LAS PRÁCTICAS CORPORALES URBANAS Y SU POSIBLE INSER- CIÓN EN LAS CLASES DE EDUCACIÓN FÍSICA EN LA ESCUELA SECUNDARIA ${ }^{1}$
}

\author{
Jorge Ricardo Saraví \\ Universidad Nacional de La Plata, Buenos Aires, Argentina \\ Tony Honorato \\ Universidade Estadual de Londrina, Londrina, Paraná, Brasil
}

\begin{abstract}
Resumen
El objetivo de este ensayo es reflexionar sobre la presencia de las prácticas corporales urbanas en la educación física escolar secundaria. Vinculadas a la cultura urbana en el tiempo libre de los jóvenes, estas prácticas desarrollan un conjunto de procesos sociales e identitarios y suelen ser comúnmente olvidadas o ignoradas cuando se planean los contenidos de la educación física en la escuela brasileña y argentina de hoy. Entre otras razones, quizás esto se debe a que ellas no están sistematizadas en toda su magnitud en la enseñanza formal y pueden ser conflictivas y/o confrontar algunos aspectos del sistema escolar.
\end{abstract}

Palabras clave: Educación Física. Prácticas corporales urbanas. Escuela secundaria. Jóvenes.

\section{Introducción}

En este ensayo propondremos algunas reflexiones vinculadas a las prácticas corporales urbanas (roller, skate, BMX, parkour, slackline, hip-hop, entre otras) y su relación con la educación física escolar. Para ello tomaremos como referencia algunos elementos de la realidad de Argentina y de Brasil.

Para comenzar queremos destacar que muchas de estas prácticas están vinculadas a la cultura urbana extra-escolar de los jóvenes. Se trata de prácticas productoras de un conjunto de procesos sociales y que a la vez generan principios educativos sin haber sido estructuradas intencionalmente para este propósito. Ellas no se limitan al espacio-tiempo de la escuela, sino que son educativas debido a sus propias dinámicas culturales de distinción social y, debido a que implican formación de valores, de intercambio de conocimientos, de transferencia de elementos objetivos y subjetivos para la producción identitaria.

Estas prácticas suelen ser comúnmente olvidadas o ignoradas cuando se planifican los contenidos anuales de la educación física en las escuelas brasileña y argentina de hoy. Entre otras razones, quizás esto se debe a que ellas no están sistematizadas en toda su magnitud en la enseñanza formal, y que pueden ser conflictivas y/o confrontar el sistema escolar.

\footnotetext{
${ }^{1}$ O presente trabalho contou com apoio financeiro da Fundação Araucária de Apoio ao Desenvolvimento Científico e Tecnológico do Estado Paraná 
En la actualidad la educación escolar no da cuenta de los modos de vida urbanos contemporáneos, en los cuales se establecen configuraciones educativas que no se limitan a la enseñanza cotidiana institucional y al aprendizaje. Para Carrano (2003), las prácticas corporales de la ludicidad también ocupan diferentes espacios educativos de las ciudades y se presentan como una forma de reconocimiento de estructuras ocultas que escapan a la institucionalización escolar y a la previsible intencionalidad pedagógica.

Existe una necesidad de repensar la escuela, en particular la enseñanza media, que intenta educar y contener a los adolescentes y jóvenes. Frecuentemente las culturas juveniles se sitúan en la vereda opuesta de los currículos escolares, cuando ellas quizás deberían afrontar el reto de la búsqueda de una mayor interacción entre la cultura escolar y la cultura producida en el mundo social de sus actores (TENTI FANFANI, 2000; ZIBAS, 2003).

En lo que concierne a la Educación Física, Hébrard (2001) apunta la existencia de numerosas prácticas cada vez más seductoras pero a la vez extrañas a la escuela, que suelen ser entendidas o pensadas como "inviables" en el contexto escolar. En esa misma línea, entendemos que en Argentina y Brasil (y quizás en América Latina en general), una pregunta clave podría ser: ¿Qué es lo que se debe enseñar en las clases de educación física? Y asociado a ello, algunas preguntas de orden didáctico: ¿Cómo enseñar esos contenidos? ¿Para qué enseñarlos? ¿Cuándo?

\section{La inclusión de las prácticas corporales urbanas en la escuela}

En los últimos años varios autores en diferentes países han indagado en las relaciones existentes y posibles entre las prácticas de aventura, radicales y urbanas. Al respecto podríamos mencionar los trabajos de Calogirou (2001), Cornejo (2004), Franco (2008), Pereira \& Armbrust (2010), Cássaro (2011), Armbrust \& Silva (2012), Pimentel, et al (2013), Saraví (2014; 2015) y Marques França et al. (2015). Los abordajes y decodificaciones con mayor fuerza en estas temáticas se han realizado a partir de las lecturas que tienen por marco teórico las Ciencias Sociales (en particular la Antropología, la Sociología y la Historia). Pero a pesar de esos estudios, consideramos que aún se carece de suficiente investigación empírica como para establecer un diálogo en profundidad entre la temática de las prácticas corporales urbanas y la Educación Física en tanto disciplina educativa. Se evidencia que estas prácticas de la ciudad aparecen en la mirada de los investigadores como un tema provocativo para comprender mejor a los jóvenes.

Según Hincapié Zapata,

Las prácticas corporales son ejecuciones corporales que se realizan con significados y propósitos propios [...] Las prácticas corporales urbanas son aquellas que se ponen en escena en la ciudad, emergen de las culturas juveniles y se muestran como una manifestación política, social, que permite mostrar una resistencia, unos rasgos identitarios particulares y una posibilidad de expresión y apropiación de los espacios de ciudad (HINCAPIE ZAPATA, 2012, p. 271).

De acuerdo Armbrust e Silva (2012), los contenidos de esa cultura corporal son pertinentes para transmitir una tradición construida históricamente y para hacer que los alumnos se apropien y se enriquezcan con ellas. Mientras tanto, deben ser renovados para la actualización y la ampliación de los significados de los alumnos, porque existen diferentes contextos, códigos y lenguajes de grupos sociales que están siendo vividos fuera de la escuela ${ }^{2}$.

\footnotetext{
${ }^{2}$ Algunos estados de la Federación de Brasil han redactado sus propias directivas curriculares en ese sentido. El Estado de Paraná (2008) prevé entre la enseñanza de contenidos algunas prácticas que suelen ser consideradas 
Desde esta perspectiva cabría preguntarse si la inclusión de este tipo de prácticas como contenidos obligatorios en el sistema escolar -, se convertiría en un obstáculo o favorecería el desarrollo de la Educación Física. ¿Hasta qué punto hoy la escuela está (o debería estar) abierta a nuevas prácticas vinculadas a la sociabilidad de los jóvenes?

A primera vista, el problema parecería ser que la Educación Física ha ignorado e ignora las prácticas corporales urbanas. Sin embargo, vale la pena preguntarse acerca de la necesidad, pertinencia y validez de su enseñanza, y respecto a cuáles serían sus fines educativos en el proceso escolar. En ese marco, antes de introducirlas en la escuela, consideramos necesario un debate amplio y profundo sobre el papel que estas prácticas tendrían en contextos educativos formalizados.

En el caso que fuese posible incluir las prácticas corporales urbanas en los currículos, se haría necesario capacitar recursos humanos para enseñarlas, dadas las dificultades institucionales (y de las políticas educativas) de hacer frente a lo no conocido y a prácticas no familiares para la tradición de la Educación Física. Entre otros obstáculos también podríamos citar, por un lado, posibles resistencias basadas en disposiciones legales poco flexibles, y por el otro, problemas de orden económico. Este último tema está relacionado con lo que a menudo señalan los profesores en referencia a la falta de recursos financieros para la adquisición de materiales destinados al uso en las clases de Educación Física, así como a la falta de infraestructura adecuada. Somos conscientes de que por ejemplo, para la práctica de $B M X$ se requieren bicicletas, para patinar se necesitan rollers, y así podríamos continuar la lista. Está claro que dichos equipos suelen ser caros (o al menos más caros que un balón de fútbol o voleibol). Asimismo a veces requieren cierto cuidado en su uso y manejo, especialmente en cuanto a términos de seguridad, lo cual requiere una cierta capacitación y/o idoneidad para la manipulación y enseñanza de cómo usar esos materiales.

Todo esto puede potencialmente dificultar la propuesta y su aplicación, pero consideramos que ello no es imposible. En Brasil se han llevado adelante experiencias tales como la introducción del slackline en escuelas de la ciudad de Joinville (Santa Catarina), así como otras iniciativas aisladas sin una orientación o sistematización vinculada a los diseños curriculares. También a modo de ejemplo, podemos destacar en Argentina la experiencia del Centro de Educación Media - CEM - № 36 de Bariloche, donde cerca de treinta jóvenes concurren dos veces por semana a un taller de skate, así como las experiencias llevadas adelante en la Escuela Número 8 de Berisso ${ }^{3}$.

A partir de lo enunciado anteriormente podríamos formular la siguiente pregunta: ¿La escuela secundaria es receptiva a nuevas prácticas y propuestas corporales? (nos referimos aquí a la escuela como institución y no a los profesionales que llevan adelante la disciplina Educación Física). Podríamos ir más lejos aún y preguntarnos: ¿Qué es lo que deben aprender los jóvenes en la escuela? ¿Deben ser formados para el ingreso a la universidad, para el mundo del trabajo, para la práctica social? Tales preguntas debieran estar en el centro de las preocupaciones educacionales que buscan proporcionar calidad, ciudadanía y futuro para la comunidad $^{4}$.

deportes "radicales" (skate; rappel; rafting; trekking; bungee jumping; surf). A pesar de ello, en general, tales contenidos no han sido luego implementados en su totalidad en todas las escuelas.

${ }^{3}$ El taller que mencionamos en el CEM 36 de Bariloche, si bien se realiza con alumnos de la escuela secundaria, funciona en un horario extraescolar. Para más detalles ver: (ANB, 2011).

${ }^{4}$ Según Tenti Fanfani (2000, p. 5), “La enseñanza media de hoy tiene un significado distinto al que tenía en el proyecto fundacional de los sistemas educativos occidentales [...] En las condiciones actuales, la llamada enseñanza media es una enseñanza "final" (un "techo") para la mayoría de la población y un momento de un proceso de formación de una minoría, que tiende a prolongarse a lo largo de toda su trayectoria vital (educación permanente)". 
Sería válido también preguntarse: ¿Cuáles son los sentidos que las prácticas corporales urbanas tienen o podrían tener para las familias de los alumnos? ¿Sería similar la posible aceptación del skate como contenido, en una escuela de elite o en una escuela que trabaja con hijos de familias socioeconómicamente desfavorecidas? Por ejemplo: ¿Qué representaciones y adaptaciones sobre la enseñanza y el aprendizaje producirían estas familias de clase alta $o$ media alta si supieran que sus hijos aprenderán hip-hop en la escuela? ¿Qué prejuicios deberían dejarse de lado para que esto sea aceptado por los sujetos del grupo familiar y de su entorno cercano?

Un proyecto de tal magnitud, que tenga como objetivo ampliar el abanico de contenidos a enseñar en la clase de Educación Física, introduciendo nuevos saberes en la escuela, debería tomar en consideración múltiples aspectos. No necesariamente dejar de lado las iniciativas pedagógicas vigentes, sino quizás brindar elementos suficientes con la intención de sensibilizar a la comunidad educativa sobre la pertinencia y el interés de tal experiencia. Esto podría facilitar la adhesión por parte de las familias y de los demás miembros de la comunidad.

Se podría además intentar establecer otra línea de reflexión que vincule la expansión y la elección de las prácticas corporales urbanas como contenidos escolares, con el tema de la calidad de la educación que se ofrece. A modo de ejemplo: una escuela que programe clases y/o lecciones de skate ¿Les brindaría otras oportunidades de educación de mayor calidad a sus estudiantes? En ciertos niveles de la sociedad, especialmente en las escuelas privadas bilingües, se suele valorar particularmente una mayor cantidad de horas de práctica de deportes y de Educación Física, por lo cual a priori el tema de la calidad parecería que pasa por una valoración cuantitativa. Pero, ¿Qué dirían las familias que llevan sus hijos a estas escuelas si el proyecto educativo pusiera énfasis en deportes alternativos o diferentes?

A partir de los estudios de Pierre Bourdieu (1990), sabemos que el valor simbólico otorgado a la elección y a la práctica de deporte, también acompaña los valores de clase social. ¿Sería viable entonces imaginar una escuela de tradición inglesa en Argentina, sin rugby o sin hockey? O mejor aún, intentemos imaginar los deportes colectivos hegemónicos (fútbol, basquetbol, voleibol, handbol) en las clases de Educación Física, en escuelas públicas y privadas brasileiras, compartiendo la carga horaria con prácticas corporales urbanas como el hiphop, parkour, roller, BMX. Difícil ejercicio imaginativo para lo que suele ser el pensamiento conservador de las instituciones escolares, pero seguramente mucho más interesante para el joven (y porque no también para el profesor, para la escuela y para la sociedad toda). Mejorar la calidad educativa no se trataría de introducir nuevas prácticas corporales en la escuela, sino también de pensar y repensar cómo se enseñan o enseñarían dichas prácticas. Quizás un camino posible es trabajar con los docentes y brindarles herramientas que les permitan repensarse a sí mismos en su accionar cotidiano y repensar los aspectos pedagógicos de la enseñanza de esos nuevos contenidos. El handbol, el voleibol, el skate, el parkour no podrían ni deberían ser enseñados de igual manera. No sólo porque son prácticas corporales distintas, con lógicas internas diferentes (PARLEBAS, 2001) ${ }^{5}$, sino también porque los públicos, los actores sociales y protagonistas son otros, los contextos sociales de aplicación no serían los mismos y las escuelas donde estas experiencias se pondrían en acción son diferentes también unas de otras. Rockwell (1995, p.15) afirma que el conocimiento escolar respecto a las escuelas se construye, y propone "reconstruir el eslabón que media entre programa y alumnos, es decir la experiencia escolar cotidiana que condiciona el carácter y el sentido de lo que es posible aprender en la escuela"

\footnotetext{
${ }^{5}$ Parlebas (2001), define el concepto lógica interna como las características principales o los rasgos pertinentes de una determinada situación motriz. 
Investigaciones sobre la cultura skater (HONORATO, 2005; SARAVÍ, 2012) muestran que el skate no tiene tradición de ser enseñado a través de los caminos pedagógicos tradicionales de una institución escolar, sino que se suelen desarrollar otros mecanismos de transmisión y aprendizaje en ámbitos no formales. Para Honorato (2005), este proceso es desrutinizador y está sustentado en cuatro bases: el relativo poder de escoger la práctica y el movimiento; el lócus de aprendizaje no es único ni permanente; las ambivalencias están permitidas; y la aparición del descontrol emocional, paradójicamente está "controlado". En su investigación sobre skate en la ciudad de La Plata, Saraví (2012) relevó la existencia de escuelitas de skate privadas, en las cuales la enseñanza no es llevada adelante por profesores de Educación Física, sino que están a cargo de skaters experimentados. En general en los spots localizados en espacios públicos ${ }^{6}$, la forma de aprender es a través de la co-enseñanza con pares, es decir que se aprende mirando al otro, escuchando y dando consejos para repetir muchas veces los movimientos y así lograr adquirir el dominio de las técnicas. Desde esta perspectiva se considera que el skate desarrollado en marcos no institucionalizados es una práctica corporal urbana relacionada al uso del tiempo libre y el ocio, donde las competiciones no son lo más importante. Al contrario, el foco en la mayoría de los casos, esta puesto en el placer, la diversión y en la libertad que les otorga dicha práctica (SARAVÍ, 2012).

Estas prácticas urbanas que por momentos se plantean o se presentan como "alternativas" y que en este texto hemos propuesto discutir como posibles nuevos contenidos escolares, quizás también podrían hacer viable un trabajo educativo interdisciplinario. A modo de ejemplo, ciertas prácticas corporales expresivas, como algunas danzas, no serían sólo patrimonio exclusivo de la Educación Física sino que permitirían articular proyectos con Música, Plástica, Historia, Ciencias Sociales.

Si consideramos la escuela como un espacio de construcción social (colectiva, consensuada), una escuela deseada y deseable, cabe interrogar si no podrían introducirse conocimientos que son significativos para los alumnos y que suelen estar relegados. Lo opuesto pareciera ser una escuela aislada socialmente, una isla en la cual no entrarían ciertas propuestas, es decir, donde no habría lugar para ciertas prácticas no consagradas por la institución educativa o no avaladas por las instancias y perspectivas tradicionales.

Sintetizando, a lo largo de nuestro escrito hemos planteado interrogantes en torno a si el skate, el roller, el parkour y otras prácticas corporales urbanas que aún no están institucionalizadas y que se encuentran ocupando el tiempo libre de los adolescentes y jóvenes, pueden entrar a las instituciones educativas o no, y por qué. Con sólo efectuar un recorrido por algunas escuelas secundarias, hablar informalmente con docentes o hacer el ejercicio de revisar documentos oficiales tales como diseños curriculares, sin dudas veremos que es difícil encontrar una respuesta clara a nuestra pregunta.

A las dificultades institucionales se suman aspectos administrativos y burocráticos, la resistencia al cambio del profesorado y el temor de las autoridades a que los alumnos/as sufran accidentes, entre los obstáculos potenciales a encontrar. Se podrían plantear aquí algunas ideas para llevar adelante propuestas de prácticas corporales urbanas. Por ejemplo: no en todas las escuelas permiten que los adolescentes y jóvenes puedan entrar con una tabla de skate bajo el brazo. Por lo tanto, un primer paso concreto sería que se permitiera la entrada a establecimientos educativos con tablas de skate, rollers y otros implementos, acción que en general suele ser no aceptada por las autoridades de las escuelas. La vicedirectora de una institución educativa de nivel secundario de la ciudad de La Plata -Argentina-, nos narraba que en su escuela dejan entrar a los jóvenes con la tabla de skate, pero que si quieren utilizarla rodando o deslizándose, inmediatamente les llaman la atención diciéndoles que eso no es posible. La

\footnotetext{
${ }^{6}$ La palabra spot es un anglicismo que designa un lugar que tiene características muy propicias y atractivas para la práctica del skate y que es elegido por sus protagonistas. Es una abreviatura de spotlight (foco de luz potente y directo que ilumina una zona pequeña, utilizado en fotografía y cine).
} 
fundamentación de ello, según la docente, sería que "no tienen lugares adecuados de práctica dentro de la escuela". Esto nos parece un primer paso, ya que no se expulsa ese objeto de alto valor simbólico - que es la tabla - sino que se permite incluirla (como elemento de significación y de identificación personal), pudiendo así los protagonistas de la práctica skater, tenerla consigo, tanto en la escuela como en el resto de su vida cotidiana.

De todas maneras, coincidimos con Calogirou (2001) cuando se pregunta: ¿Cuál sería el sentido de querer introducir el skateboard en la escuela, considerando que fuera de ella el contexto social que debería hacer crecer esa práctica deportiva no lo hace? Esto lo vemos cuando los gestores del poder político municipal no dan apoyo o retrasan obras de construcción de pistas de skate o skateparks, cuando las fuerzas del orden público expulsan a quienes llevan adelante prácticas corporales urbanas de las veredas o explanadas de edificios céntricos, o cuando simplemente los vecinos protestan de manera airada por los ruidos que producen estos jóvenes (SARAVÍ, 2012).

Un problema clave es como entendemos la ciudadanía y a partir de ello, la definición de quienes pueden ser considerados ciudadanos. Cuando algunos vecinos expresan que estas nuevas prácticas "invaden" el espacio público de las urbes, y denuncian a los jóvenes que las llevan adelante con la excusa o motivo de las supuestas molestias que se generan con sus acciones, sonidos y movimientos, cabe preguntarse: ¿Cómo se define o se decide quiénes son los que tienen derecho a la ciudad y quiénes no? ¿Cómo se construye el "nosotros", y a partir de allí, donde se ubican o posicionan los otros que son excluidos? Sin dudas hay muchos elementos para reflexionar al respecto, desde una perspectiva de derechos de ciudadanía.

\section{Consideraciones finales}

Nuestro eje de análisis en este ensayo ha sido poner en debate algunas relaciones posibles entre prácticas corporales urbanas y escuela. En esa misma perspectiva, nos hemos preguntado si estos conocimientos pueden pasar a formar parte de los contenidos de la Educación Física escolar, y como sería ese proceso (en caso de que sea posible).

Algunos pasos, tímidos pero claros, han comenzado a ser dados en algunos ámbitos escolares. Si bien a corto plazo surgen demasiados interrogantes, creemos que la incorporación de ciertas prácticas corporales será un proceso inevitable. Esta introducción podría colaborar a superar la fosa entre la cultura escolar y las culturas juveniles (TENTI FANFANI, 2000). Sin embargo, el problema es muy complejo y está lejos de ser resuelto. Por eso será necesario contar con el apoyo de quienes llevan adelante las políticas públicas educativas, de los propios profesores/as (así como de sus órganos de representatividad y de gestión), y también de los alumnos/as y miembros de la comunidad escolar. La sociedad en su conjunto deberá analizar y buscar soluciones para este problema que presenta facetas muy diversas.

En una reflexión de carácter más general consideramos que es necesario pensar y analizar si se tienen en cuenta las necesidades, deseos y expectativas de nuestros jóvenes en las escuelas secundarias (sean estas referidas a la práctica de determinados deportes o disciplinas corporales, o a cuestiones del arte, de la música, de los pasatiempos, de los juegos, etc.). En particular, y referido a la Educación Física, son necesarios replanteos respecto al lugar que se le otorga al disfrute del cuerpo y del movimiento en nuestra escuela actual. Es necesario considerar como por ejemplo se piensa que los chicos puedan ir con agrado a una clase de Educación Física y que no falten porque el campo de deportes es muy lejos o porque la clase es aburrida ( $u$ otras posibles razones que suelen ser escuchadas en boca de nuestros alumnos adolescentes). ¿De qué manera se podría contemplar que los alumnos puedan encontrar placer al moverse con las prácticas escogidas para ser desarrolladas y enseñadas en clase? Algunos de estos contenidos vienen siendo enseñados casi sin grandes variaciones desde hace varias décadas. 
La Educación Física, como materia de enseñanza escolar que intenta ser puesta en valor, tiene varios desafíos pendientes a futuro. Uno de ellos lo hemos planteado a lo largo del artículo: la incorporación de prácticas corporales urbanas como contenidos a enseñar.

Finalizaremos este texto con una pregunta, que quizás sea al mismo tiempo una afirmación y una propuesta. La posibilidad de incluir algunas prácticas corporales que en las últimas décadas han crecido exponencialmente en ámbitos urbanos y que son pasión de miles de jóvenes: ¿Produciría un cambio significativo en la Educación Física escolar? Agregar más contenidos ampliaría el abanico de prácticas corporales enseñadas y, por lo tanto, podría nutrir de nuevas experiencias a nuestros estudiantes, brindando posibilidades de actualización y arrojando una bocanada de frescura. Pero una acumulación de contenidos novedosos no necesariamente significaría una revisión de modos de enseñar, ni tampoco implicaría otorgarle otro lugar al sujeto como protagonista de la clase. La inclusión de nuevos contenidos puede contribuir para mejorar la disciplina y enriquecerla. Pero la Educación Física puede continuar siendo tan tradicional como lo ha sido, si no realiza una profunda renovación de muchos de sus postulados. Esperamos que las reflexiones que hemos intentado compartir aquí con los lectores y lectoras permitan aportar en la búsqueda de nuevos caminos.

\title{
AS PRÁTICAS CORPORAIS URBANAS E SUA POSSÍVEL INSERÇÃO NAS AULAS DE EDUCAÇÃO FÍSICA NA ESCOLA SECUNDÁRIA
}

\section{Resumo}

O objetivo deste ensaio é refletir sobre a presença das práticas corporais urbanas na Educação Física do ensino médio. Ligada à cultura urbana no tempo livre dos jovens, essas práticas desenvolvem um conjunto de processos sociais e identitários e são comumente esquecidas ou ignoradas quando são planejados os conteúdos anuais de Educação Física para a escola no Brasil e na Argentina. Entre outras razões, talvez isso seja porque elas não estão sistematizadas em toda a sua magnitude para a educação formal e podem ser conflitantes e/ou confrontar alguns aspectos do sistema escolar.

Palavras-chave: Educação Física. Práticas corporais urbanas. Escola secundária. Jovens.

\section{URBAN CORPOREAL PRACTIES ANS THEIR POSSIBLE INSERTION INTO PHYSICAL EDUCATION CLASSES IN SECONDARY EDUCATION}

\begin{abstract}
The objective of this essay it to reflect upon the presence of corporal practices within physical education classes during high school. Linked to the urban culture of youths free time, these practices develop a set of social and identifying processes, and are commonly forgotten or ignored during annual curriculum planning for physical education for schools in Brazil and Argentina. Among other reasons, perhaps this may be because such practices are not standardized in all their magnitude for use in formal education and can therefore be conflicting and/or clash with some aspects of the school system.
\end{abstract}

Keywords: Physical Education, Urban body practices, Secondary school, Young people. 


\section{Referências}

ANB. Skate, el deporte de autosuperación que atrajo a los alumnos del CEM 36. Disponible en: < http://www.anbariloche.com.ar/noticias/2014/06/11/42869-skate-el-deporte-deautosuperacion-que-atrajo-a-los-alumnos-del-cem-36> Acceso en: 15 out. 2016.

ARMBRUST, I.; SILVA, S. A. P. S. Pluralidade cultural: os esportes radicais na Educação Física escolar. Movimento, Porto Alegre, v. 18, n. 01, p. 281-300, jan./mar. 2012. Disponible en: 〈http://www.seer.ufrgs.br/Movimento/article/view/14937>

Acceso en: 20 dez. 2013.

BOURDIEU, P. ¿Cómo se puede ser deportista? En Sociología y Cultura, México, 1990.

CALOGIROU, C. Las actividades deportivas. Debates sobre estudios e innovaciones: E1 ejemplo del skateboard. Los nuevos deportes en la escuela. Revista Stadium, n. 176, Buenos Aires, 2001.

CARRANO, P. C. R. Juventudes e cidades educadoras. Rio de Janeiro: Editora Vozes, 2003.

CÁSSARO, E. R. Atividades de aventura: aproximações preliminares na rede municipal de ensino de Maringá. 2011. Monografia (Especialização em Educação Física na Educação Básica) - Universidade Estadual de Londrina, Londrina, 2011.

CORNEJO, M. Las prácticas deportivas informales de los jóvenes chilenos: una nueva forma de identidad colectiva. Revista Visiones, n. 5, p. 7-10, 2004.

FRANCO, L. C. P. Atividades físicas de aventura na escola: uma proposta pedagógica nas três dimensões do conteúdo. 2008. Dissertação (Mestrado em Educação Física) - Universidade Estadual Paulista, Rio Claro/SP, 2008.

HÉBRARD, A. Las actividades deportivas. Debates sobre estudios e innovaciones: Introducción al coloquio. Revista Stadium, n. 176, Buenos Aires, 2001.

HINCAPIE ZAPATA, A. La escuela, un lugar para la apropiación de las prácticas corporales urbanas de danza en la Comuna 13 de la ciudad de Medellín, Colombia. Revista Estudios pedagógicos, Valdivia, v. 38, n. especial, p. 267-291, 2012. Disponible en: $<$ http://www.scielo.cl/scielo.php?script=sci_arttext\&pid=S071807052012000400015\&lng=es\&nrm=iso> Acceso en: 8 mayo. 2016.

HONORATO, T. A tribo skatista e a instituição escolar: o poder escolar em uma perspectiva sociológica. 2005. Dissertação (Mestrado em Educação) - Universidade Metodista de Piracicaba, 2005.

MARQUES FRANÇA, R.; SILVA BOZZ, A. C.; FURIHATA, M.; BARGERI ROSA, G.; SCHIAROLLI, G. Esportes radicais (ER) na escola: fundamentações e experimentações. Actas del $7^{\circ}$ Congreso CONPEF. "Aula de Educação Física: espaço de diálogo, tensões, res- 
significações e compromissos". p. 1-12, maio. 2015. Disponible en: <http://www.conpef.com.br/anteriores/2015/artigos/30.pdf > Acceso en: 15 out. 2016.

PARANÁ. Diretrizes curriculares da educação básica - educação física - do Estado do

Paraná. Curitiba: SEED, 2008. Disponible en:

<http://www.nre.seed.pr.gov.br/cascavel/arquivos/File/edfisica.pdf> Acceso en: 20 dez. 2013.

PARLEBAS, P. Juegos, deportes y sociedad. Léxico de praxiología motriz. Barcelona: Editorial Paidotribo, 2001.

PEREIRA, D. W.; ARMBRUST, I. Pedagogia da aventura: os esportes radicais, de aventura e de ação na escola. Jundiaí/SP: Editora Fontoura, 2010.

ROCKWELL, E. De huellas, bardas y veredas: una historia cotidiana en la escuela. La escuela cotidiana. México: Fondo de Cultura Económica, 1995.

SARAVÍ, J. R. Skate, espacios urbanos y jóvenes en la ciudad de La Plata. 2012. Tesis de Maestría (en Educación Corporal) - Facultad de Humanidades y Ciencias de la Educación, Universidad Nacional de La Plata, 2012.

.Los estudios de prácticas corporales urbanas desde la perspectiva de las Ciencias Sociales. Aportes al campo de la Educación Física. EZEQUIEL, C.; GERARDO, F.; NÉSTOR, H.; AYELÉN, M.; OSVALDO, R.; MARTÍN, U. (Org.). Prácticas de la Educación Física. La Plata: Edición de la Facultad de Humanidades y Ciencias de la Educación, UNLP. Colección Colectiva y monográfica, n. 2, 2014. Disponible en:

<http://www.libros.fahce.unlp.edu.ar/index.php/libros/catalog/view/45/61/383-1 > Acesso en: 12 out. 2016.

.Prácticas corporales urbanas y Educación Física: nuevos desafíos y posibilidades. TORRES, A. F. R.; TORO, J. R. T.; ALVARES, D. M. (Org.). Educación Física y Ciencias del Deporte en Latinoamérica: un enfoque multidisciplinario. Ecuador: Editado por el Instituto de Investigación y Posgrado, Facultad de Cultura Física, de la Universidad Central del Ecuador y el Centro de Investigación y Desarrollo, Guayaquil, 2015. Disponible en:

$<$ http://media.wix.com/ugd/54b18d_ff05c1b321f8499bb1aea0f9a15ecac7.pdf > Acceso en: 15 out. 2016.

TENTI FANFANI, E. Culturas juveniles y cultura escolar. IIPE-Buenos Aires. Serie Documentos del IIPE, mayo 2000. Disponible en:

<http://www.buenosaires.iipe.unesco.org/sites/default/files/panel4_fanfani.pdf.> Acesso en: 28 jan. 2014.

ZIBAS, D. La reforma de la enseñanza media en Brasil en los años '90': críticas y nuevas perspectivas. Actas del Encuentro "Desafíos de la enseñanza secundaria en Francia y los países del Cono Sur". Ciudad de Buenos Aires, septiembre de 2003. Disponible en: <http://www.bnm.me.gov.ar/giga1/documentos/EL001245.pdf > Acceso en: 28 jan. 2014. 
Endereço para correspondência:

tonyhonoratu@gmail.com

Tony Honorato

Universidade Estadual de Londrina

Rodovia Celso Garcia Cid, Km 380, s/n

Campus Universitário

Londrina - PR, 86057-970 